\title{
Biological and chemical diversity of cytotoxin-producing symbiotic marine fungi in intertidal zone of Dalian
}

\author{
ZHANG Yi ${ }^{1,2,3 *}$, MU Jun ${ }^{2 *}$, FENG Yan $^{2}$, LI HeNan ${ }^{2} \&$ DONG XueWei ${ }^{2}$ \\ ${ }^{1}$ School of Life Science and Biotechnology, Dalian University of Technology, Dalian 116024, China; \\ ${ }^{2}$ School of Environmental and Chemical Engineering, Dalian Jiaotong University, Dalian 116028, China; \\ ${ }^{3}$ Key Laboratory of Experimental Marine Biology, Institute of Oceanology, Chinese Academy of Sciences, Qingdao 266071, China
}

Received May 2, 2012; accepted July 23, 2012; published online September 16, 2012

\begin{abstract}
In this study, the biological and chemical diversity of 8 symbiotic marine fungal strains, with strong cytotoxicity against brine shrimp larvae, were investigated by nucleotide sequencing, morphology and cluster analysis of HPTLC fingerprint. These strains were identified by ITS rDNA sequencing, phylogenic analysis, and morphology to be Hypocrea lixii, Chaetomium globosum, Aspergillus fumigatus, Asp. clavatus and Alternaria sp. Their differences in secondary metabolites were shown by cluster analysis of digitalized colors of HPTLC spots, a newly developed method, which produced a similar dendrogram with that of ITS cluster analysis. Furthermore, this method can fully display intraspecific differences and even the remarkable difference in Aspergillus strains which goes beyond the boundary between genera. Their biological-chemical diversity may be the basis of their potent cytotoxicity and implies their potential in producing diversified antitumor or pesticidal constituents.
\end{abstract}

symbiotic marine fungi, cytotoxicity, diversity, rDNA sequencing, morphology, fingerprint

Citation: Zhang Y, Mu J, Feng Y, et al. Biological and chemical diversity of cytotoxin-producing symbiotic marine fungi in intertidal zone of Dalian. Chin Sci Bull, 2013, 58: 2290-2297, doi: 10.1007/s11434-012-5442-2

Symbiotic fungi live on the surface or in the inner tissue of their hosts. Some terrestrial symbiotic fungi have been found to produce toxins or anti-feedants to protect their hosts from predators and grazers [1]. Some of these compounds can be used as antitumor or pesticidal agents. Symbiotic marine fungi have been isolated from seaweeds, sponges, corals, mangroves and sea grasses, also showing taxonomicall diversity and producing numerous active compounds [2].

The intertidal coastline of Dalian possesses diversified natural and artificial habitats and also high biodiversity of marine plants, invertebrates, and microorganisms. In our screening for useful cytotoxins from local symbiotic marine fungi using brine shrimp lethality test, a widely used bifunctional preliminary screening model to discover antitumor drugs and pesticides from the sea [3-5], eight strains with potent activities were discovered. Herein, we report the

*Corresponding authors (email: hubeizhangyi@163.com; mujun1971@126.com) study on the biological and chemical diversity of these bioactive strains by ITS rDNA sequence analysis, morphology, and metabolite fingerprinting using a new cluster method.

\section{Materials and methods}

\subsection{Bioactive strains under investigation}

The eight symbiotic fungal strains, both epiphytes and endophytes, were isolated from the marine flora and fauna samples using the method previously reported [6]. The samples were collected from the intertidal zone of Fujiazhuang beach $\left(121^{\circ} 36^{\prime} 13.82^{\prime \prime} \mathrm{E}, 38^{\circ} 48^{\prime} 36.66^{\prime \prime} \mathrm{N}\right)$ in Dalian City, China, in October of 2008 and May of 2009. These strains were statically fermented for $30 \mathrm{~d}$ in $200 \mathrm{~mL}$ of PSB (potato sucrose broth) containing 2\% natural sea salt at $28^{\circ} \mathrm{C}$. Mycelia were extracted with methanol and fermentation broth was extracted by ethyl acetate. The two extracts were combined to obtain crude organic extract, 
which was finally dissolved in $3 \mathrm{~mL}$ of methanol after rotary evaporation [6]. A total of 51 strains were screened for their remarkable cytotoxicity against brine shrimp larvae in the following bioassay.

The brine shrimp (Artemia parthenogenetica) larvae were hatched and collected using the method similar to that by Micheal et al. [7] and Lu et al. [8]. Fungal extracts and controls were respectively added into the microplates, and then dried in vacuum oven at room temperature. Afterwards, $200 \mu \mathrm{L}$ of instar II-III A. parthenogenetica nauplii suspension containing 20-30 vivid larvae was added into each well. Then the microplates were incubated under fluorescent lamp in the incubator at $28^{\circ} \mathrm{C}$ for $24 \mathrm{~h}$ without cover. Pure water and methanol were respectively used as blank controls; taxol, adriamycin, and trichlorphon at final concentration of $5 \mu \mathrm{g} / \mathrm{mL}$ were used as positive controls. The results were observed and counted under a binocular dissecting microscope. The corrected average lethality rate of each sample was calculated according to Abbott Formula [9]. The bioassay consisted of 4 rounds of screenings with serially reduced dosage of extract and incubation time to select the strongest cytotoxin-producing fungal strains. For the four screenings, they were $40 \mu \mathrm{L} /$ well for $24 \mathrm{~h}, 5 \mu \mathrm{L} /$ well for $24 \mathrm{~h}$, $1 \mu \mathrm{L} /$ well for $24 \mathrm{~h}$, and $1 \mu \mathrm{L} /$ well for $4 \mathrm{~h}$, respectively.

The information about the origin, crude extract content, and bioactivity of the eight strains is listed in Table 1.

\subsection{Molecular taxonomy}

DNA extraction of the strongest active fungal strains was performed using the Plant Genomic DNA Kit DP305 (Tiangen) according to the manufacturer's protocol. PCR was then performed using TaKaRa Ex Taq polymerase (TaKaRa) and the fungal universal primer pair ITS1 and ITS4, in a Takara PCR Thermal cycler Dice TP600 with the method of White et al. [10]. Then the PCR product mixture was analyzed by DNA electrophoresis on agarose gel, purified using TaKaRa DV805A Agarose Gel DNA Purification
Kit, and sequenced by an ABI PRISMTM 3730XL DNA sequencer $(\mathrm{TaKaRa})$ with primer ITS1. The sequence data had been submitted to and deposited at GenBank with the accession numbers shown in Table 2.

The ITS1-5.8 S-ITS2 (internal transcribed spacer, ITS) rDNA sequences were used to search the GenBank database with the BlastN 2.2.19+ algorithm for the closest matches in the ITS1-5.8 S-ITS2 rDNA sequences of known species. Sequences were aligned with representative fungal ITS1-5.8 S-ITS2 rDNA sequences using Clustal X (version 1.81); a neighbour-joining phylogenetic tree was constructed using the MEGA 4.0 [11,12].

\subsection{Morphological characterization}

Morphological characterization of the fungal isolates was carried out according to standard taxonomic key including colony diameter, texture, color, and the dimensions as well as the morphology of hyphae and conidia [13].

\subsection{Cluster analysis of HPTLC fingerprint}

For the strongest active strains, $1 \mu \mathrm{L}$ of each extract was applied on high-performance thin-layer chromatographic plates (HPTLC silica gel 60 F254, Merck, Darmstadt, Germany) with a capillary. Then the HPTLC plate was developed twice by a mixture of $\mathrm{CHCl}_{3} / \mathrm{MeOH}(20: 1$, v/v). After air drying, the spots of the fungal metabolites on the plate were photographed under UV lamp at 254 and $365 \mathrm{~nm}$, and then scanned after coloration by $\mathrm{H}_{2} \mathrm{SO}_{4}$-anisaldehyde agent at $105^{\circ} \mathrm{C}$ for $2 \mathrm{~min}$.

To display the relationship and difference in secondary metabolism between these strains quantitatively, the metabolite fingerprints, i.e. the spots' colors on the HPTLC plates, were digitalized in the following section and analyzed by cluster method. Since TLC plate after chemical colorization gave more information, it was chosen for cluster analysis instead of UV TLC images. Firstly, the area between the

Table 1 The origin, crude extract yield and bioactivity of the 8 fungal strains ${ }^{\text {a) }}$

\begin{tabular}{|c|c|c|c|c|c|c|c|}
\hline \multirow{2}{*}{ Strain number } & \multirow{2}{*}{ Host } & \multirow{2}{*}{ Type } & \multirow{2}{*}{$\begin{array}{l}\text { Crude extract content } \\
\text { in samples }\left(\mathrm{mg} \mathrm{mL}^{-1}\right)\end{array}$} & \multicolumn{4}{|c|}{ Lethal rates of four rounds of brine shrimp lethality test (\%) } \\
\hline & & & & $1 \mathrm{st}$ & 2nd & $3 \mathrm{rd}$ & 4th \\
\hline DLEN2008003 & Gracilaria lemaneiformis & endo- & 43.2 & $100 \pm 0$ & $100 \pm 0$ & $100 \pm 0$ & $18 \pm 12$ \\
\hline $6-\mathrm{N}$ & Chondrus ocellatus & endo- & 60.6 & $100 \pm 0$ & $100 \pm 0$ & $88 \pm 11$ & $5 \pm 8$ \\
\hline DLEN2008004 & Sargassum thunbergii & endo- & 109.1 & $100 \pm 0$ & $100 \pm 0$ & $96 \pm 3$ & $100 \pm 0$ \\
\hline 13-F1 & S. kjellmanianum & epi- & 71.7 & $100 \pm 0$ & $100 \pm 0$ & $100 \pm 0$ & $97 \pm 4$ \\
\hline $13-\mathrm{F} 2$ & S. kjellmanianum & epi- & 44.0 & $100 \pm 0$ & $100 \pm 0$ & $85 \pm 9$ & $95 \pm 5$ \\
\hline $11-\mathrm{N} 1$ & Scytosiphon lomentarius & endo- & 69.6 & $100 \pm 0$ & $100 \pm 0$ & $100 \pm 0$ & $99 \pm 2$ \\
\hline DLEN2008006 & Reniera japonica & endo- & 57.1 & $100 \pm 0$ & $100 \pm 0$ & $100 \pm 0$ & $0 \pm 0$ \\
\hline DLEN2008010 & R. japonica & endo- & 67.9 & $100 \pm 0$ & $100 \pm 0$ & $100 \pm 0$ & $27 \pm 10$ \\
\hline
\end{tabular}

a) The dosage of extracts and effect time for the 4 rounds of screenings were $40 \mu \mathrm{L} \times 24 \mathrm{~h}, 5 \mu \mathrm{L} \times 24 \mathrm{~h}, 1 \mu \mathrm{L} \times 24 \mathrm{~h}$ and $1 \mu \mathrm{L} \times 4 \mathrm{~h}$, respectively. epi-: epiphytic; endo-: endophytic. 
Table 2 The results of molecular and morphological identification of the active strains

\begin{tabular}{|c|c|c|c|c|c|}
\hline Strain number & ITS length (bp) & Accession number & Closest reference strains & Identity (\%) & Macroscopic and microscopic identification \\
\hline DLEN2008003 & 581 & HQ156781 & Hypocrea lixii GQ328858 & 100 & Hypocrea sp. \\
\hline DLEN2008004 & 578 & GU266272 & Hypocrea lixii FJ442645 & 99 & Hypocrea sp. \\
\hline DLEN2008010 & 580 & HQ149775 & Hypocrea lixii FJ412025 & 99 & Hypocrea sp. \\
\hline $6-\mathrm{N}$ & 541 & GU244529 & Chaetomium globosum FJ772001 & 99 & Chaetomium sp. \\
\hline 13-F1 & 565 & GU266273 & Aspergillus fumigatus GQ169480 & 100 & Aspergillus sp. \\
\hline $13-\mathrm{F} 2$ & 554 & HQ149772 & Aspergillus fumigatus GU205082 & 99 & Aspergillus sp. \\
\hline DLEN2008006 & 567 & GU266275 & Aspergillus clavatus GU183170 & 99 & Aspergillus sp. \\
\hline \multirow[t]{5}{*}{$11-\mathrm{N} 1$} & 532 & HQ149771 & Alternaria alternate GU797144 & 100 & Alternaria sp. \\
\hline & & & Alternaria longipes AB470902 & 100 & \\
\hline & & & Alternaria brassicae FJ869872 & 100 & \\
\hline & & & Alternaria mali AY154683 & 100 & \\
\hline & & & Alternaria tenuissima AB369499 & 100 & \\
\hline
\end{tabular}

baseline and the terminal line was equally divided into forty zones by horizontal lines. Secondly, a copy of the HPTLC plate image was opened in ACDSee software (version 3.1), by which each zone of each strain was cut out and saved as a new picture file. Then the new files were opened in Adobe Photoshop software and the eyedropper tool was used to select the main and representative color of the zones carefully. The hexadecimal code for the selected color can be read from the color picker. These codes are of optical implication. For white color, the number is the largest value FFFFFF; while for black color, the number is the smallest value 000000. For example, for zones 16-18 (Rf 0.3740.442) of strain 13-F1, the color code for the pink spot was B83152. These hexadecimal codes can be transformed into decimal codes. For B83152, it was 12071250. Finally, the data, i.e., the decimal codes of zones 1-40 of each strain, were studied by SPSS software's (Version 13.0) Q-type hierarchical clustering method to compute the squred Euclidean distance and infer the dendrogram with its default settings.

\section{Results}

In the bioassay, strains 13-F1, 13-F2, DLEN2008006, 11N1, DLEN2008003, DLEN2008004, DLEN2008010, and $6-\mathrm{N}$ all showed lethality rates (LRs) of $100 \%$ in the first two rounds of screening and LRs over $85 \%$ in the third round (dosage: $1 \mu \mathrm{L} /$ well, equivalent to $3.2-8.2 \mu \mathrm{g} / \mathrm{mL}$ for different samples; time: $24 \mathrm{~h}$ ), which were comparable to or even stronger than the positive controls $(86.9 \%$ for taxol, $37.4 \%$ for adriamycin, and $88.7 \%$ for trichlorphon at $5 \mu \mathrm{g} / \mathrm{mL}$ in $24 \mathrm{~h}$ ) and much stronger than the remaining 43 strains (data not shown). Four of them showed extremely strong and acute toxicity against brine shrimp larvae with LRs of 95\%$100 \%$ in the last round (Table 1). These facts indicated that these strains may be able to produce some powerful and interesting cytotoxins which would be useful in new drug development. Since the constituents of bioactive products are closely related with systematics of microorganisms, these strains were further investigated with respect to their taxonomy by nucleotide sequence analysis, morphology, and secondary metabolite fingerprints to understand their metabolic potential and diversity as cytotoxin producers.

\subsection{Molecular and morphological taxonomy}

First, the eight strongest strains were primarily characterized to be the members of different genera: Aspergillus, Alternaria, Hypocrea, and Chaetomium, respectively, according to their morphological characteristics of colonies, hyphae, and spores. Some of these features are easy to recognize, such as the actinomorphic conidiophore for Aspergillus, the dark septate clavate conidia for Alternaria, the greygreen colonies with rapid horizontal growth for Hypocrea, and the hairly perithecia for Chaetomium. They were further identified by molecular analysis on their ITS1-5.8S-ITS2 ribosomal DNA sequences with the length of 532-581 bp (Table 2).

The BLAST alignment of their ITS1-5.8S-ITS2 (simplified as ITS) rDNA sequences with the data stored in GenBank showed that strains DLEN2008003, DLEN2008004 and DLEN2008010 were 99\%-100\% similar to the reference strains of the species Hypocrea lixii, 6-N 99\% similar to Chaetomium globosum, 13-F1 and13-F2 99\%-100\% similar to Aspergillus fumigatus, and DLEN2008006 99\% similar to Asp. clavatus, respectively. In a phylogenic tree constructed by N-J algorithm (Figure 1), these strains were all placed on the corresponding clades with the reference species from GenBank (degree of confidence: 93\%-100\%), while branched from other related species of the same genera 


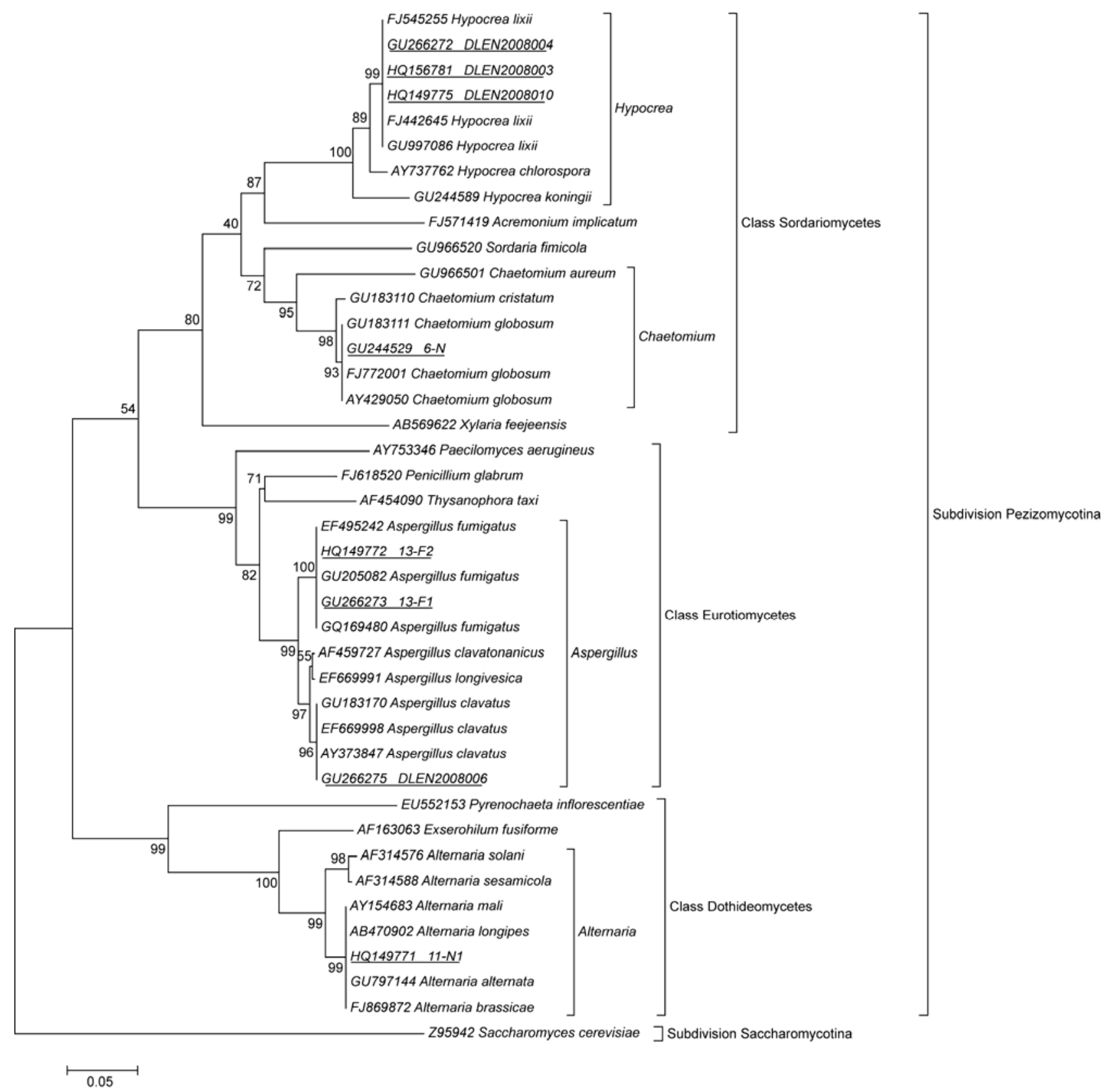

Figure 1 Neighbour-Jointing tree of ITS1-5.8S-ITS2 rDNA sequences of the top eight isolates (underlined), compared with sequences obtained from GenBank. Bootstrap values (expressed as percentages of 1000 replicates) are given at the nodes. The scale on the bottom indicates the number of substitutions per nucleotide. The Genbank accession numbers are listed before each isolate or reference strains.

(supported by 1000 bootstrap replicates).

As for strain 11-N1, the BLASTN revealed that it was highly similar to several small-spored species of genus $\mathrm{Al}$ ternaria in GenBank with ITS rDNA's identities of $100 \%$, including Alt. alternata, Alt. brassicae, Alt. longipes and Alt. mali and so on. In the phylogenic N-J tree (Figure 1), the 11-N1 isolate was also placed on the same clade with the above species (degree of confidence: 99\%), while clearly branching from the large-spored group of this genus, e.g., Alt. solani and Alt. sesamicola. However, using only ITS sequence analysis, it was difficult to discriminate the high affinity species in small-spored group of genus Alternaria [14]. More professional morphological identification is needed to determine it to species level.

\subsection{Analysis of secondary metabolites}

HPTLC analysis revealed that these strongest active strains produced highly diverse metabolites as reflected by the spots under UV light or after chemical coloration (Figure 2). Furthermore, their profiles of products were quite different on genus and species levels and even within the same species 


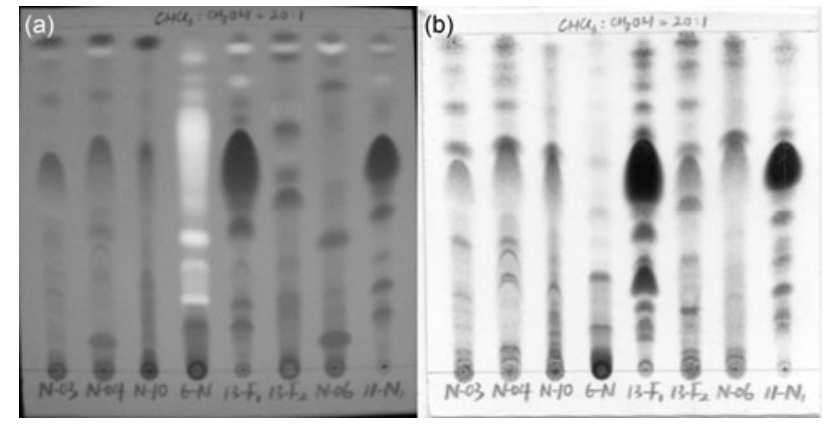

Figure 2 HPTLC analysis of the eight strongest strains (developed using Chloroform: Methanol=20:1 (v/v) twice). (a) Under UV light at 254 and $365 \mathrm{~nm}$; (b) coloration by $\mathrm{H}_{2} \mathrm{SO}_{4}$-anisaldehyde agent (the serial number of some strain were abbreviated, e.g. DLEN2008003 was simplified as N-03).

as shown by direct observation as well as by cluster analysis of fingerprint.

In the dendrogram of secondary metabolites (Figure 3), strains of genera Hypocrea, Chaotomium and Alternaria showed clear branches from each other; the relationship between them was consistent with the genetic phylogenic tree (Figure 1). Such relationship was also intuitively reflected by the disparate spots in the metabolites of 6-N (Chaetomium globosum) and 11-N1 (Alternaria sp.) in comparison with those of Hypocrea lixii strains DLEN2008003, DLEN2008004, and DLEN2008010. 6-N produced rich yellow compounds emitting white fluorescence under UV light, which became light orangish after coloration by $\mathrm{H}_{2} \mathrm{SO}_{4}$ anisaldehyde agent. 11-N1 produced rich colorful spots, e.g. vivid violet $(R f 0.58)$, pink (0.42) and purple (0.18) spots.

However, the three Aspergillus strains (Asp. fumigatus 13-F1 and 13-F2 and Asp. clavatus DLEN2008006) appeared not to be taxonomically restricted and were distributed among different genera as shown by their close relationship with Alternaria strain 11-N1 and Hypocrea lixii strains, respectively. Even though the strains 13-F1 and 13-F2 belong to the same species and were also isolated from the same host Sargassum kjellmanianum, the dendrogram showed a remote relationship between them; their TLC image also displayed quite different constituents in

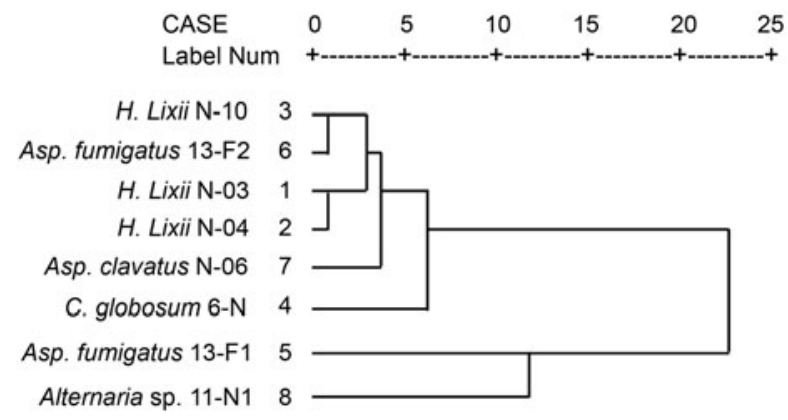

Figure 3 Cluster analysis of HPTLC fingerprint of the eight strongest strains by SPSS (the serial numbers of some strain were abbreviated, as shown in Figure 2). high, middle, and low polar sections. This phenomenon for members of genus Aspergillus might be due to the variation on strain level and their powerful potential in the production of diverse secondary metabolites [2].

The secondary metabolite variation on strain level was also observed in $H$. lixii strains DLEN2008003, DLEN2008004, and DLEN2008010 from three different hosts. They were divided into two clades in dendrogram and their high polar constituents at $R f$ value of $0-0.42$ were quite different. These variations on strain level will be useful in discovering diverse bioactive metabolites.

For strain 6-N, large-scale fermentation, extraction, chromatographic fraction, and activity tracing were preliminarily investigated in this study. The spots emitting white fluorescence at $R_{\mathrm{f}}$ of $0.46-0.77$ and $0.18-0.41$ as well as strong polar non-fluorescent constituents with $R_{\mathrm{f}}$ of 0 were found to be the main representative active components, which revealed the structural diversity of potential active compounds. Further purification and structure elucidation are underway.

To sum up, the analysis of secondary metabolism of these strongest active strains showed that they are highly chemically diverse and have the potential to produce active cytotoxins.

\section{Discussion}

\subsection{Relationship between systematics and biological potential}

As indicated by taxonomy, the symbiotic fungal strains with the strongest cytotoxicity primarily come from genera Hypocrea and Aspergillus and secondarily from Chaetomium and Alternaria. On class level, they belong to three different classes: Sodariomycetes, Eurotiomycetes and Dothideomycetes in the subdivision Pezizomycotina.

According to the reviews of Saleem et al. [15] and Blunt et al. [16-19], there are totally 80 marine fungal strains from 32 species as well as 4 unidentified strains that were reported to produce cytotoxic compounds in 2000-2009 worldwide. Among the former group, 79 strains in 31 species come from the subdivision Pezizomycotina of Ascomycota and only one strain in Rhizopus is from basal fungi occupying relatively lower position in the life tree of fungi. So far there is no report on Basidiomycota. In the cytotoxinproducing Pezizomycotina, 39 strains are reported from 4 species in Eurotiomycetes, 33 strains from 20 species in Sodariomycetes, and 7 strains from 7 species in Dothideomycetes. It seems that marine Aspergillus (20 strains reported) and Penicillium (15 strains reported) in Eurotiomycetes have the greatest potential in cytotoxin production. The members of the group with the second largest potential mostly come from Sodariomycetes, containing Trichoderma (4 strains), Chaetomium (3 strains), Gliocladium (4 strains), Gymnascella (3 strains), etc. It is interesting to note that half 
of all the cytotoxin-producing Sodariomycetes species were from order Hypocreales. The relationship between systematics and bioactivity or cytotoxic compounds among the relevant genera/species is investigated in detail as follows.

Genus Aspergillus is known for its powerful secondary metabolic potential. On marine Aspergillus alone, there have been a large number of documents about producer strains of antitumor compounds with highly diverse structural types such as polyketides, peptides, alkaloids, terpenoids and lipids [20-24]. The reported producers include Asp. glaucus, Aspergillus sp. CNC-120, Asp. fumigatus, Asp. ustus, Asp. terreus and others from different hosts or sediments. Also reported is a marine algous Asp. oryzae producing indoloditerpenes against brine shrimp larvae [25]. Based on these reports, it may be easy to understand the highly heterogeneous HPTLC fingerprints and bioactivity of our three Aspergillus strains.

As we already know, terrestrial Hypocrea (anamorph Trichoderma) species are important biocontrol organisms against plant pathogens. Also, some marine Hypocrea strains from hosts, seawater or sediments are reported to possess larvicidal or antitumor activities, including $T$. koningii against blowfly larvae and strains which inhibit different cancer cell lines, e.g., a Trichoderma sp., T. reesei, T. longibrachiatum and T. virens [26-30]. Their diverse cytotoxic products are reported to include $\alpha$-aminoisobutyric acid, dipeptides, cyclotetrapeptide, peptides, peptaibols, cyclopentenone and polyketide derivatives. Maybe some novel or known compounds in these or some other families can form the substantial basis for bioactivity of our cytotoxic Hypocrea strains.

Chaetomium globosum has been isolated from marine red alga Polysiphonia urceolata, green alga Ulva pertusa and fish Mugil cephalus. It is interesting that the three different strains also produced quite different families of antitumor compounds, i.e., benzaldehyde derivatives, cytochalasans and azaphilones [31-34].

A marine Alternaria tenuis from alga is also found to produce antitumor isocoumarin [35]. Besides, quite a few terrestrial Alternaria strains also give relevant reports. For example, tenuazonic acid and its derivative produced by Alt. tenius and plant endophytic Alternaria No. 28 showed pesticidal effects in field experiments and significant cytotoxic activity in brine shrimp bioassay, respectively [36,37]. Also reported were Alt. porri producing alterporriol $\mathrm{F}$ and plant endophytic Alternaria sp. producing 5 compounds against cancer cells $[38,39]$.

As revealed by these documentary surveys, not only the cytotoxic activity but also the diverse active compounds from these taxa are closely related with systematics, which implies the great potential in producing diverse cytotoxins of genera Hypocrea, Aspergillus, Alternaria and Chaetomium. There are also reports on larvicidal activity (against brine shrimp) and related compounds. Our HPTLC analysis clearly indicated that the eight larvicidal strains can produce highly diverse secondary metabolites. Furthermore, the preliminary tracing of strain $6-\mathrm{N}^{\prime}$ cytotoxins also showed the activity of different components with diverse structure-related properties. Therefore, these strains may be of importance in the search for novel cytotoxins to develop new antitumor agents and pesticides.

\subsection{Cluster analysis of rDNA sequence and metabolite fingerprint in fungal taxonomy}

For the 8 active strains, their ITS rDNA sequences and secondary metabolic fingerprints were both analyzed by cluster method in this study. The result showed that the ITS1-5.8SITS2 rDNA sequences were quite conservative on species level and well consistent with morphological taxonomy. They can be used as a convenient tool by nonprofessional researchers in fungal identification. However, it cannot entirely replace classic morphological taxonomy when dealing with some species with highly similar or completely identical ITS1-5.8S-ITS2 rDNA sequence such as Alternaria sp. Considering that different secondary metabolites can be represented by different colors of spots on TLC plates with specific $R_{\mathrm{f}}$ values after chemical colorization, the cluster analysis of HPTLC fingerprints was also applied in present study. The results showed that this method can give similar relationship as by genetic analysis and it can further exhibit the difference on intraspecific level. However, the strains in some species had extraordinarily strong secondary metabolism potential, e.g. Aspergillus spp., which may show clear branches or even cross the gaps between different genera. So this method may be taken as an assistant tool for intraspecific taxonomy and may also be suitable for intragenus taxonomy of highly similar species in some genera such as Alternaria.

Though cluster analyses of TLC fingerprint have been reported for plant and lichen taxonomy, previous reports mainly adopted principal component analysis (PCA) for some known or similar compounds from closely related plant samples, for which binary codes " $0 / 1$ " were used to represent the absence or presence of specific metabolites [40-43]. Obviously, this method is not suitable for relationship analysis of entirely unknown and rich constituents of microorganisms, which are highly diverse taxonomically and chemically. The method in the present study resolved this question better by transforming the colors of different polar zones into standard hexadecimal codes to produce serial data for hierarchical cluster analysis. This method can be used as an assistant chemical taxonomical tool to analyze different microorganisms and macroorganisms. Although commercial scanner and manual color selection were used in this study due to the limitation of experimental conditions, satisfactory dendrogram was still obtained. If this method can be further improved and performed by automatic thinlayer scanner, it has the potential to be widely applied in chemical taxonomy. 


\section{Conclusions}

In the selection of potential microbial strains for bioactive lead compounds' discovery and production, systematics by molecular genetic, morphological and chemtaxonomical methods can be a useful and rational guide. Based on ITS rDNA sequence, morphology, cluster analysis of HPTLC fingerprints, literature survey as well as preliminary toxins tracing, eight larvicidal symbiotic fungal strains were discovered to be diverse species/strains both biologically and chemically, with good prospect in cytotoxin production for medicinal or agrochemical purposes. Besides, the newly developed method of cluster analysis of HPTLC fingerprints by spot color digitalization and SPSS was able to obtain similar dendrogram with genetic phylogenic tree in the relationship analysis of fungal strains and show more detailed difference in secondary metabolism. This method may find extensive applications in chemotaxonomy after some modifications.

This work was supported by the National Natural Science Foundation of China (20902009), China Postdoctoral Science Foundation (2011M500051), the Key Laboratory of Experimental Marine Biology, Institute of Oceanology, Chinese Academy of Sciences (kf201009) and the Scientific and Technological Fund of Dalian (2008J23JH041).

1 Tan R X, Zou W X. Endophytes: A rich source of functional metabolites. Nat Prod Rep, 2001, 18: 448-459

2 Bugni T S, Ireland C M. Marine-derived fungi: A chemically and biologically diverse group of microorganisms. Nat Prod Rep, 2004, 21: 143-163

$3 \mathrm{Hu}$ Z Y, Liu S Z, Huang H, et al. A rapid screening assay for insecticidal antibiotic produced by marine actinomycetes (in Chinese). Mar Sci Bull (Haiyang Tongbao), 2000, 19: 36-41

4 Carballo J L, Hernández-Inda Z L, Pérez P, et al. A comparison between two brine shrimp assays to detect in vitro cytotoxicity in marine natural products. BMC Biotechnol, 2002, 2: 17-21

5 Xiong L, Li J, Kong F. Streptomyces sp. 173, an insecticidal micro-organism from marine. Lett Appl Microbiol, 2004, 38: 32-37

6 Zhang Y, Mu J, Feng Y, et al. Broad-spectrum antimicrobial epiphytic and endophytic fungi from marine organisms: Isolation, bioassay and taxonomy. Mar Drug, 2009, 7: 97-112

7 Michael A S, Thompson C G, Abramovitz M. Artemia salina as a test organism for a bioassay. Science, 1956, 123: 464

8 Lu Y, Chen S Y, Fu C X. Testing method of brine shrimp toxicity and the testing kit. PRCh Patent. CN200510050702.1 (laid open) 2005-07-14

9 Abbott W S. A method of computing the effectiveness of an insecticide. J Econ Entomol, 1925, 18: 265-267

10 White T J, Bruns T, Lee S, et al. Amplification and direct sequencing of fungal ribosomal RNA genes for phylogenetics. In: Innis M A, Gelfand D H, Sninsky J J, et al., eds. PCR Protocols: A Guide to Methods and Applications. New York: Academic Press, 1990. 315322

11 Thompson J D, Gibson T J, Plewniak F, et al. The CLUSTALX windows inter-face: Flexible strategies for multiple sequence alignment aided by quality analysis tools. Nucleic Acids Res, 1997, 25: 4876-4882

12 Tamura K, Dudley J, Nei M, et al. MEGA4: Molecular evolutionary genetics analysis (MEGA) software version 4.0. Mol Biol Evol, 2007, 24: 1596-1599

13 Ainsworth G C, Frederick K S, Alfred S S. A taxonomic review with keys: Ascomycetes and Fungi Imperfecti. In: Ainsworth G C, Sparrow F K, Sussman A S, eds. The Fungi: An Advanced Treatise, Volume IV(A). New York and London: Academic Press, 1973. 621

14 Wang H K, Zhang T Y, Zhang M. Application of sequencing of 5.8S rDNA, ITS1 and ITS2 on identification and classification of Alternaria at species level (in Chinese). Mycosystema (Junwu Xitong), 2001, 20: 168-173

15 Saleem M, Ali M S, Hussain S, et al. Marine natural products of fungal origin. Nat Prod Rep, 2007, 24: 1142-1152

16 Blunt J W, Copp B R, Hu W P, et al. Marine natural products. Nat Prod Rep, 2008, 25: 35-94

17 Blunt J W, Copp B R, Hu W P, et al. Marine natural products. Nat Prod Rep, 2009, 26: 170-244

18 Blunt J W, Copp B R, Munro M H, et al. Marine natural products. Nat Prod Rep, 2010, 27: 165-237

19 Blunt J W, Copp B R, Munro M H, et al. Marine natural products. Nat Prod Rep, 2011, 28: 196-268

20 Du L, Zhu T J, Fang Y C, et al. Aspergiolide A, a novel anthraquinone derivative with naphtho[1,2,3-de]chromene-2,7-dione skeleton isolated from a marine-derived fungus Aspergillus glaucus. Tetrahedron, 2007, 63: 1085-1088

21 Wang F Z, Fang Y C, Zhu T J, et al. Seven new prenylated indole diketopiperazine alkaloids from holothurianderived fungus Aspergillus fumigatus. Tetrahedro, 2008, 64: 7986-7991

22 Toske S G, Jensen P R, Kauffman C A, et al. Aspergillamides A and $\mathrm{B}$ : Modified cytotoxic tripeptides produced by a marine fungus of the genus Aspergillus. Tetrahedron, 1998, 54: 13459-13466

23 Liu H B, Edrada-Ebel R A, Ebel R, et al. Drimane sesquiterpenoids from the fungus Aspergillus ustus isolated from the marine sponge Suberites domuncula. J Nat Prod, 2009, 72: 1585-1588

24 Parvatkar R R, D'Souza C, Tripathi A, et al. Aspernolides A and B, butenolides from a marine-derived fungus Aspergillus terreus. Phytochemistry, 2009, 70: 128-132

25 Qiao M F, Ji N Y, Liu X H, et al. Indoloditerpenes from an algicolous isolate of Aspergillus oryzae. Bioorg Med Chem Lett, 2010, 20: 5677-5680

26 Landreau A, Pouchus Y F, Sallenave-Namont C, et al. Combined use of LC/MS and a biological test for rapid identification of marine mycotoxins produced by Trichoderma koningii. J Microbiol Methods, 2002, 48: 181-194

27 You J, Dai H, Chen Z, et al. Trichoderone, a novel cytotoxic cyclopentenone and cholesta-7,22-diene-3 $\beta, 5 \alpha, 6 \beta$-triol, with new activities from the marine-derived fungus Trichoderma sp. J Ind Microbiol Biotechnol, 2010, 37: 245-252

28 Sun Y, Tian L, Huang J, et al. Trichodermatides A-D, novel polyketides from the marine-derived fungus Trichoderma reesei. Org Lett, 2008, 10: 393-396

29 Ruiz N, Wielgosz-Collin G, Poirier L, et al. New Trichobrachins, 11-residue peptaibols from a marine strain of Trichoderma longibrachiatum. Peptides, 2007, 28: 1351-1358

30 Garo E, Starks C M, Jensen P R, et al. Trichodermamides A and B, cytotoxic modified dipeptides from the marine-derived fungus Trichoderma virens. J Nat Prod, 2003, 66: 423-426

31 Wang S, Li X M, Teuscher F, et al. Chaetopyranin, a benzaldehyde derivative, and other related metabolites from Chaetomium globosum, an endophytic fungus derived from the marine red alga Polysiphonia urceolata. J Nat Prod, 2006, 69: 1622-1625

32 Cui C M, Li X M, Li C S, et al. Cytoglobosins A-G, cytochalasans from a marine-derived endophytic fungus, Chaetomium globosum QEN-14. J Nat Prod, 2010, 73: 729-733

33 Yasuhide M, Yamada T, Numata A, et al. Chaetomugilins, new selectively cytotoxic metabolites, produced by a marine fish-derived Chaetomium species. J Antibiot (Tokyo), 2008, 61: 615-622

34 Yamada T, Muroga Y, Tanaka R. New azaphilones, seco-chaetomugilins A and D, produced by a marine-fish-derived Chaetomium globosum. Mar Drugs, 2009, 7: 249-257

35 Huang Y F, Li L H, Tian L, et al. Sg17-1-4, a novel isocoumarin from a marine fungus Alternaria tenuis $\mathrm{Sg} 17-1$. J Antibiot (Tokyo), 2006, 59: 355-357 
36 Zhang Q W, Luo K. Preliminary research on biocontrol of Aphis gossypii and Tetranychus cinnabarinus by tenuazonic acid (in Chinese). Plant Prot (Zhiwu Baohu), 1990, 16: 34-35

37 Qin J C, Zhang Y M, Hu L, et al. Cytotoxic metabolites produced by Alternaria no.28, an endophytic fungus isolated from Ginkgo biloba. Nat Prod Commun, 2009, 4: 1473-1476

38 Phuwapraisirisan P, Rangsan J, Siripong P, et al. New antitumour fungal metabolites from Alternaria porri. Nat Prod Res, 2009, 23: 1063-1071

39 Aly A H, Edrada-Ebel R, Indriani I D, et al. Cytotoxic metabolites from the fungal endophyte Alternaria $\mathrm{sp}$. and their subsequent detection in its host plant Polygonum senegalense. J Nat Prod, 2008, 71:
972-980

40 Ye W H, Kuang H X, Wang Z Y, et al. Studies on the population differentiation of Aralia elata (Miq.) seem. (II) Cluster analysis on the chemical constituents (in Chinese). Bull Bot Res (Zhiwu Yanjiu), 1999, 19: 207-212

41 Chai R J, Wang Y L. Chemical study of the foliose lichens of five ordors in Xinjiang. For By Prod Special China (Zhongguo Lin Fu Techan), 2007, 3: 26-29

42 Zhao H R, Wang R P, Wang Y, et al. The application of cluster analysis in the authentication and taxonomy of Lonicera spp. and counterfeit species (in Chinese). Shanxi J Trad Chin Med (Shanxi Zhongyi), 2005, 26: 72-73

Open Access This article is distributed under the terms of the Creative Commons Attribution License which permits any use, distribution, and reproduction in any medium, provided the original author(s) and source are credited. 